\title{
Maternal serum markers of lipid metabolism in relation to neonatal anthropometry
}

\author{
NS Boghossian ${ }^{1}, \mathrm{P}$ Mendola ${ }^{2}, \mathrm{~A} \mathrm{Liu}^{2}, \mathrm{C}$ Robledo $^{3}$ and EH Yeung ${ }^{2}$
}

\begin{abstract}
OBJECTIVE: The objective of this study is to examine associations between lipids (high-density lipoprotein, low-density lipoprotein, total cholesterol, triglycerides and lipoprotein (a)) measured on average three time points during pregnancy and neonatal anthropometrics.

STUDY DESIGN: Stored samples from a preeclampsia trial measured as part of a case-control study from five US centers (1992 to $1995)$ were used. The sample included women without pregnancy complications $(n=136)$ and cases of gestational diabetes ( $n=93)$, abnormal glucose tolerance (AGT; $n=76)$, gestational hypertension $(n=170)$ and preeclampsia $(n=177)$. Linear regression and linear mixed-effects models estimated adjusted associations between lipids and birth weight $z$-score, ponderal index (PI), length and head circumference.

RESULTS: Among women without complications, cross-sectional associations between total cholesterol measured at different gestational ages increased PI 2.23 to $2.55 \mathrm{~kg} \mathrm{~m}^{-3}$ per-unit increase in cholesterol. HDL was inversely associated with birth length $\left(\beta^{\prime} s=-2.21\right.$ and $\left.-2.56 \mathrm{~cm}\right)$. For gestational hypertension, triglycerides were associated with birth weight $z$-score $(\beta$ 's $=0.24$ to 0.31$)$. For preeclampsia, HDL was associated with lower birth weight $z$-scores ( $\beta$ 's $=-0.49$ and -0.82 ). Women with gestational diabetes or AGT had inconsistent associations. Examining the level changes across pregnancy, each $0.0037 \mathrm{mmol}^{-1}$ increase in $\mathrm{HDL}$ was associated with decreased birth weight $z$-score $(\beta=-0.22)$, length $(\beta=-0.24 \mathrm{~cm})$ and head circumference $(\beta=-0.24 \mathrm{~cm})$, whereas each $0.028 \mathrm{mmol} \mathrm{I}^{-1}$ increase in triglycerides was associated with increased birth weight $z$-score $(\beta=0.13)$ and head circumference $(\beta=0.19 \mathrm{~cm})$.

CONCLUSIONS: Although associations varied by complications, in general, growth-promoting fuels such as total cholesterol and triglycerides were associated with increased neonatal size, whereas high HDL was associated with smaller size. Maternal HDL that failed to decrease over pregnancy was associated with smaller neonate size.
\end{abstract}

Journal of Perinatology (2017) 37, 629-635; doi:10.1038/jp.2017.22; published online 23 March 2017

\section{INTRODUCTION}

Inappropriate fetal growth, often assessed by birth weight, has an important effect on the future risk of coronary heart disease, hypertension, and type 2 diabetes. ${ }^{1}$ Although the impact of hyperglycemia in pregnancy, particularly in overt gestational diabetes, on fetal growth is well documented, ${ }^{2-4}$ studies examining associations between other maternal fuels and newborn size, however, have been limited. The majority of the studies examined lipid biomarkers measured at one time point during pregnancy, while restricting the study sample to diabetic pregnancies, nondiabetic pregnancies with positive diabetic screens or uncomplicated pregnancies. ${ }^{5-11}$ Findings from these studies have been inconsistent. We sought to understand whether the heterogeneity in previous findings were due to having different timing of specimens, different case mix group of women with and without complications or the specific measure of neonatal size evaluated. Furthermore, studies did not have multiple measures from both before and after the clinical diagnosis of these conditions. Lipid biomarkers, if proven useful, may also be relatively easy to implement in clinical settings, as hospital laboratories are already equipped to measure lipids for the purpose of cardiovascular screening in non-pregnant populations. We examined whether maternal circulating fuels, measured on average at three time points during pregnancy, influenced neonatal size stratifying by common pregnancy complications (that is, gestational diabetes, abnormal glucose tolerance (AGT), gestational hypertension, preeclampsia or no reported pregnancy complications) and adjusting for other maternal factors known to impact fetal growth.

\section{MATERIALS AND METHODS}

Study population

The study population was derived from the Calcium for Preeclampsia Prevention trial, a randomized double-blind clinical trial (1992 to 1995) conducted among 4589 healthy nulliparous women with singleton pregnancies and without preexisting hypertension or proteinuria across five US centers, to examine the impact of calcium supplementation on the incidence and severity of preeclampsia. ${ }^{12}$ Women were randomized at a gestational age between 13 weeks 0 days and 21 weeks 6 days as determined from the earliest obstetrical ultrasound..$^{12}$ Details of the study have been published elsewhere. ${ }^{12}$ The trial showed no effect of calcium on the risk or severity of preeclampsia. ${ }^{13}$ The final sample size included 3667 women after excluding women who were lost to follow-up $(n=283)$, those who had a pregnancy loss $(n=49)$, had an infant with a chromosomal abnormality $(n=1)$ or had no blood collected during the baseline screening visit or misdated samples $(n=589) .{ }^{14}$ We measured lipids as

\footnotetext{
${ }^{1}$ Department of Epidemiology and Biostatistics, Arnold School of Public Health, University of South Carolina, Columbia, SC, USA; ${ }^{2}$ Division of Intramural Population Health Research, Eunice Kennedy Shriver National Institute of Child Health and Human Development, National Institutes of Health, Bethesda, MD, USA and ${ }^{3}$ Department of Behavioral and Community Health, University of North Texas, Fort Worth, TX, USA. Correspondence: Dr EH Yeung, Division of Intramural Population Health Research, Eunice Kennedy Shriver National Institute of Child Health and Human Development, National Institutes of Health, 6710B Rockledge Drive, Room 3122, Bethesda, MD 20817, USA.

E-mail: yeungedw@mail.nih.gov

Received 16 November 2016; revised 15 January 2017; accepted 31 January 2017; published online 23 March 2017
} 
part of a nested case-control study, to examine biomarkers associated with risk of pregnancy complications. ${ }^{15}$ Given the use of existing de-identified samples, this study was exempted from Institutional Review Board review and approved by the National Institutes of Health's Office of Human Subjects Research

\section{Pregnancy complications strata}

The 'normal' group free of pregnancy complications $(n=136)$ consisted of a random group of women who were normoglycemic, normotensive, without proteinuria and who delivered a term, not small for gestational age infant. The pregnancy complication strata included women with gestational diabetes mellitus (GDM; $n=93)$, AGT (that is, having an initial positive diabetic screen followed by a normal glucose tolerance test) $(n=76)$, gestational hypertension $(n=170)$ and preeclampsia $(n=177)$. Women with more than one pregnancy complication were included in each group accordingly. Detailed definitions of preeclampsia and gestational hypertension diagnosis are published elsewhere. ${ }^{13,14,16}$ Briefly, preeclampsia was defined as pregnancy-associated hypertension, a diastolic blood pressure $\geqslant 90 \mathrm{~mm} \mathrm{Hg}$ on two different occasions 4 to $168 \mathrm{~h}$ apart and proteinuria, characterized as one of the following: a urine dipstick of at least $1+\left(30 \mathrm{mg} \mathrm{dl}^{-1}\right)$ on two occasions ( 4 to $168 \mathrm{~h}$ apart), a urine dipstick of at least $2+\left(100 \mathrm{mg} \mathrm{dl}^{-1}\right)$, a protein:creatinine ratio $\geqslant 0.35$ or a $24 \mathrm{~h}$ urine specimen with $\geqslant 300 \mathrm{mg}$ of protein. Gestational hypertension used the same cutoffs of diastolic blood pressure with no proteinuria. Gestational diabetes was defined according to the American Diabetes Association 1997 criteria, with a plasma glucose $>200 \mathrm{mg} \mathrm{dl}^{-1}$ $1 \mathrm{~h}$ after $50 \mathrm{~g}$ glucose challenge test without an oral glucose tolerance test or a $50 \mathrm{~g}$ glucose challenge test result $>140 \mathrm{mg} \mathrm{dl}^{-1}$ at $1 \mathrm{~h}$ and two abnormal values on the $3 \mathrm{~h} 100 \mathrm{~g}$ oral glucose tolerance test. ${ }^{17}$ The oral glucose tolerance test cutoffs were $\geqslant 95 \mathrm{mg} \mathrm{dl}^{-1}$ fasting, $\geqslant 180 \mathrm{mg} \mathrm{dl}^{-1}$ at $1 \mathrm{~h}, \geqslant 155 \mathrm{mg} \mathrm{dl}^{-1}$ at $2 \mathrm{~h}$ and $\geqslant 140 \mathrm{mg} \mathrm{dl}^{-1}$ at $3 \mathrm{~h}$. AGT was defined as those who failed the $50 \mathrm{~g}$ glucose challenge test but had a subsequent normal oral glucose tolerance test.

\section{Covariates}

At enrollment, women self-reported their age, race/ethnicity (Non-Hispanic White, Non-Hispanic Black, Hispanic or other), smoking status, education, insurance status and marital status. Body mass index (BMI) was calculated based on weight measured during the screening visit before 21 weeks' gestation and self-reported height.

\section{Outcomes}

The baby's birth weight, length and head circumference were abstracted from medical records. Ponderal index (PI) was calculated as (birth weight $\left.(\mathrm{g}) \times 100 /(\text { crown heel length }(\mathrm{cm}))^{3}\right)$. Birth weight $z$-score was defined according to US tables that account for race, parity, gestational age and sex of the infant. ${ }^{18}$ Large for gestational age (LGA) was defined as birth weight above the 90th percentile for gestational age. ${ }^{18}$ Gestational age was verified by ultrasound data from before enrollment.

\section{Lipid measurement}

Non-fasting maternal blood samples were taken at the baseline visit before randomization (any time before 21 weeks and 6 days of gestation) and on average twice during follow-up. Serum samples were frozen and stored at $-70{ }^{\circ} \mathrm{C}$ for 17 to 21 years. Low- and high-density lipoprotein (LDL and $\mathrm{HDL}$ ), total cholesterol, triglycerides and lipoprotein (a) (Lp(a)) were measured by Roche Modular P chemistry analyzer (Roche Diagnostics, Indianapolis, IN, USA) with coefficients of variation $<4 \%$. At higher levels of $\operatorname{Lp}(\mathrm{a})\left(\geqslant 47 \mathrm{mg} \mathrm{dl}^{-1}\right)$, the coefficient of variation was $6.5 \%$. In total, 1640 serum samples from 595 women $(n=39$ women with 1 sample, $n=123$

Table 1. Characteristics of study participants by pregnancy complication group in the CPEP Study

\begin{tabular}{|c|c|c|c|c|c|c|}
\hline Characteristic & $\begin{array}{l}\text { Total } \\
\mathrm{N}=595\end{array}$ & $\begin{array}{l}\text { Controls } \\
\mathrm{N}=136\end{array}$ & $\begin{array}{c}\text { Gestational } \\
\text { diabetes }^{\mathrm{a}} \\
\mathrm{N}=93\end{array}$ & $\begin{array}{l}A G T^{b} \\
\mathrm{~N}=76\end{array}$ & $\begin{array}{l}\text { Preeclampsia }^{c} \\
\mathrm{~N}=177\end{array}$ & $\begin{array}{c}\text { Gestational } \\
\text { hypertension }^{\mathrm{d}} \\
\mathrm{N}=170\end{array}$ \\
\hline \multicolumn{7}{|l|}{ Race/ethnicity n (\%) } \\
\hline Non-hispanic white & $220(37.0)$ & 47 (34.6) & $51(54.8)$ & $30(39.5)$ & $53(29.9)$ & $62(36.5)$ \\
\hline Non-hispanic black & $285(47.9)$ & $63(46.3)$ & $32(34.4)$ & $32(42.1)$ & $95(53.7)$ & $88(51.8)$ \\
\hline Married $n(\%)$ & $143(24.1)$ & $30(22.1)$ & $35(37.6)$ & $20(26.3)$ & $37(20.9)$ & 37 (21.9) \\
\hline Less than high school $n(\%)$ & $246(41.5)$ & $60(44.1)$ & $32(34.4)$ & $29(38.2)$ & 76 (43.4) & 74 (43.5) \\
\hline No private insurance $n$ (\%) & $535(89.9)$ & $118(86.8)$ & $79(85.0)$ & $65(85.5)$ & $166(93.8)$ & $158(92.9)$ \\
\hline BMI $\left(\mathrm{kg} \mathrm{m}^{-2}\right)$ & $27.1(6.3)$ & $25.5(5.6)$ & $29.9(6.5)$ & $25.8(4.9)$ & $28.0(6.4)$ & $27.1(6.6)$ \\
\hline Current smoker $n(\%)$ & $73(12.3)$ & $10(7.4)$ & $20(21.5)$ & 14 (18.4) & $22(12.4)$ & $18(10.6)$ \\
\hline Male infant $n(\%)$ & $296(49.8)$ & $63(46.3)$ & $50(53.8)$ & $34(44.7)$ & 91 (51.4) & $88(51.8)$ \\
\hline Birth weight $(g)$ & 3163 (692) & $3314(445)$ & 3424 (583) & $3248(453)$ & $2853(818)$ & $3244(716)$ \\
\hline SGA $n(\%)$ & $55(9.2)$ & $0(0.0)$ & $4(4.3)$ & $7(9.2)$ & $33(18.6)$ & $14(8.2)$ \\
\hline LGA $n(\%)$ & $52(8.7)$ & $9(6.6)$ & $15(16.1)$ & $6(7.9)$ & $9(5.1)$ & $19(11.2)$ \\
\hline Preterm birth < 37 weeks $n(\%)$ & 87 (14.6) & $6(4.4)$ & $7(7.5)$ & $2(2.6)$ & $59(33.3)$ & $16(9.4)$ \\
\hline Gestational age at delivery (weeks) & $38.5(2.5)$ & $39.3(1.52)$ & 38.6 (1.7) & 39.1 (1.3) & $37.3(3.0)$ & 38.7 (2.6) \\
\hline $\begin{array}{l}\text { Gestational age at collection of first } \\
\text { sample (weeks) }\end{array}$ & $15.9(3.2)$ & $15.6(2.6)$ & $16.0(2.7)$ & $15.7(2.6)$ & $16.2(4.5)$ & $15.8(2.4)$ \\
\hline $\begin{array}{l}\text { Gestational age at collection of second } \\
\text { sample (weeks) }\end{array}$ & $27.7(3.1)$ & $28.3(3.3)$ & $27.7(3.0)$ & $27.7(3.0)$ & $27.6(3.5)$ & $27.4(2.7)$ \\
\hline $\begin{array}{l}\text { Gestational age at collection of third } \\
\text { sample (weeks) }\end{array}$ & $35.8(1.7)$ & $36.2(0.9)$ & $35.7(1.5)$ & $36.1(0.96)$ & $35.3(2.4)$ & $35.9(1.6)$ \\
\hline
\end{tabular}

Abbreviations: AGT, abnormal glucose tolerance; BMI, body mass index; CPEP, Calcium for Preeclampsia Prevention; LGA, large for gestational age; PI, ponderal index; SGA, small for gestational age. Figures are means (s.d.) unless otherwise specified. Data missing for: married: 1, education: 2, Pl: 32, length: 32, head circumference: 31 . ${ }^{\mathrm{a}}$ Of the 93 women with gestational diabetes, 14 also had preeclampsia and 22 also had gestational hypertension. ${ }^{\mathrm{b}}$ Of the 76 women with AGT, 5 also had preeclampsia and 16 also had gestational hypertension. ${ }^{\circ}$ Of the 177 women with preeclampsia, 14 also had gestational diabetes. ${ }^{\mathrm{d}} \mathrm{Of}$ the 170 women with gestational hypertension, 22 also had gestational diabetes. 
Table 2. Means of lipid biomarkers across gestation by pregnancy complication group in the CPEP Study

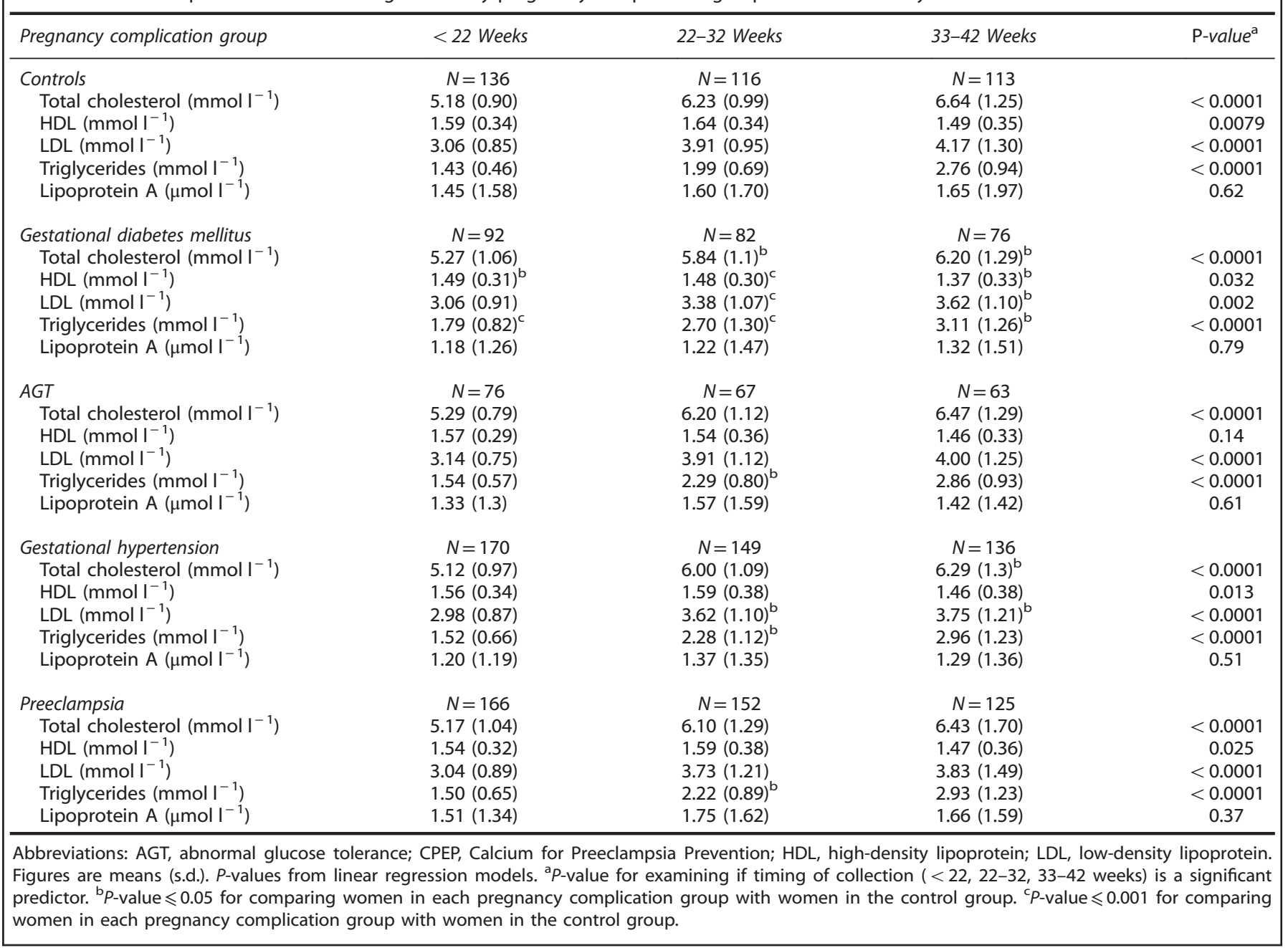

with 2 samples, $n=378$ with 3 samples, $n=54$ with 4 samples and $n=1$ with 5 samples) were sent for measurement of lipids.

\section{Statistical methods}

We described first maternal and neonatal characteristics, represented by mean (s.d.) or $n(\%)$ as appropriate, by pregnancy complication group. We also examined the lipid biomarker means across gestation $(<22,22$ to 32 and 33 to 42 weeks) by pregnancy complication group. Linear regression models were used to examine whether significant differences existed in the lipid biomarker means across the three measurement time points during pregnancy and when comparing women in each pregnancy complication group to women in the control group. Correlations between lipid measurements and maternal BMI were estimated by Pearson's correlation coefficient. We subsequently examined associations between each maternal lipid biomarker and neonatal anthropometry crosssectionally at $<22$ weeks, 22 to 32 weeks and 33 to 42 weeks using linear regression models adjusting for maternal ethnicity/race (NonHispanic white, non-Hispanic black and Hispanic or other), current smoker (yes/no) and BMI (continuous), stratifying by each pregnancy complication to eliminate potential biases in the association between maternal metabolic factors and newborn anthropometrics. Women with preeclampsia, for example, may have growth-restricted infants due to placental insufficiency rather than metabolic factors. We additionally adjusted for gestational age for models evaluating $\mathrm{Pl}$, length and head circumference as outcomes. We also evaluated the odds ratios and 95\% confidence intervals (Cls) of being born LGA in association with maternal hypertriglyceridemia (triglyceride levels $>75$ th percentile value based on all serum measures across gestation) cross-sectionally at $<22$ weeks, 22 to 32 weeks and 33 to 42 weeks using logistic regression analyses and adjusting for the same above variables. Using linear mixed-effects models, we subsequently assessed the lipid measurements across pregnancy by examining how the lipid trajectory (individual slopes) is associated with the examined newborn anthropometrics. No adjustments were made for multiple testing but we mark in tables associations with Bonferroni corrected $P$-values of $<0.01$ in place of $<0.05$ for statistical significance due to evaluating 5 lipid biomarkers that may have overlapping pathways $(0.05 / 5)$. All analyses were conducted using SAS version 9.3 (SAS Institute, Cary, NC, USA) and R (version 3.1, The R Foundation for Statistical Computing, Vienna, Austria).

\section{RESULTS}

Table 1 shows the characteristics of the mothers and their infants. On average, the women were 21 years old and with a BMI of $27.1 \mathrm{~kg} \mathrm{~m}^{-2}$ at the screening visit. Around $50 \%$ were Black and the majority were not married (76\%) and had no private insurance (90\%). Triglycerides and $\mathrm{Lp}(\mathrm{a})$ were weakly correlated with maternal BMI ( $r=0.10, P<0.001 ; r=0.08, P=0.001$, respectively), whereas no significant correlations were detected for LDL and total cholesterol. Maternal BMI was inversely related to HDL-cholesterol $(r=-0.12, P<0.001)$.

Mean levels of all the examined biomarkers differed significantly across pregnancy, except for HDL and Lp(a) (Table 2). HDL was higher at the baseline measurement $<22$ weeks in 
Table 3. Associations between maternal lipid biomarkers and neonatal anthropometrics by pregnancy complication group at three measurements across gestation in the CPEP Study

\begin{tabular}{|c|c|c|c|}
\hline & $\begin{array}{l}\text { Baseline } \\
<22 \text { weeks }\end{array}$ & $\begin{array}{c}\text { Second } \\
22-32 \text { weeks }\end{array}$ & $\begin{array}{c}\text { Third } \\
\text { 33-42 weeks }\end{array}$ \\
\hline \multicolumn{4}{|l|}{ Normal } \\
\hline \multicolumn{4}{|l|}{ Total cholesterol } \\
\hline $\mathrm{PI}\left(\mathrm{kg} \mathrm{m}^{-3}\right)$ & $2.23(0.24,4.22)$ & $2.36(0.38,4.33)$ & $2.55(0.87,4.22)^{1}$ \\
\hline $\mathrm{PI}\left(\mathrm{kg} \mathrm{m}^{-3}\right)$ & $6.77(1.13,12.4)$ & & \\
\hline Length, $\mathrm{cm}$ & $-2.56(-4.43,-0.68)^{1}$ & & $-2.21(-4.22,-0.19)$ \\
\hline \multicolumn{4}{|l|}{ LDL } \\
\hline $\mathrm{PI}\left(\mathrm{kg} \mathrm{m}^{-3}\right)$ & & & $2.05(0.49,3.61)$ \\
\hline Length $(\mathrm{cm})$ & & & $-0.52(-1.02,-0.009)$ \\
\hline \multicolumn{4}{|l|}{ Triglycerides } \\
\hline Birth weight $z$-score & & & $0.19(0.004,0.37)$ \\
\hline Birth weight z-score & & & $0.21(0.001,0.42)$ \\
\hline \multicolumn{4}{|l|}{ Gestational hypertension } \\
\hline \multicolumn{4}{|l|}{ Total cholesterol } \\
\hline Birth weight $z$-score & $0.22(0.032,0.41)$ & & \\
\hline Head circumference $(\mathrm{cm})$ & $0.32(0.033,0.60)$ & & $0.24(0.008,0.47)$ \\
\hline \multicolumn{4}{|l|}{ LDL } \\
\hline Head circumference $(\mathrm{cm})$ & $0.35(0.040,0.67)$ & & \\
\hline \multicolumn{4}{|l|}{ Triglycerides } \\
\hline Birth weight $z$-score & $0.31(0.018,0.60)$ & $0.29(0.11,0.48)^{1}$ & $0.24(0.066,0.41)^{1}$ \\
\hline Length $(\mathrm{cm})$ & & & $0.45(0.10,0.80)$ \\
\hline \multicolumn{4}{|l|}{ Preeclampsia } \\
\hline \multicolumn{4}{|l|}{ Total cholesterol } \\
\hline Birth weight $z$-score & & & $0.16(0.034,0.30)$ \\
\hline Length $(\mathrm{cm})$ & & $0.30(0.033,0.57)$ & \\
\hline
\end{tabular}

comparison with the final measurement at 33 to 42 weeks, whereas $L p(a)$ did not show any changes across gestation. Compared with women without pregnancy complications, women with GDM had lower levels of total cholesterol, HDL and LDL but higher levels of triglycerides, whereas women with gestational hypertension had lower levels of total cholesterol and LDL but higher levels of triglycerides. Women with preeclampsia or AGT in comparison with women without pregnancy complications had similar levels of all the lipid biomarkers, except for triglycerides.

Table 3 shows only the significant associations between maternal lipid biomarkers and neonatal anthropometrics by pregnancy complication group at the three time point measurements across gestation. In general, total cholesterol, LDL and triglycerides were associated with greater growth, whereas HDL was associated with less growth. However, some variability in the associations was observed between women with different pregnancy complications depending on the type of lipid biomarker, timing of the measurement and anthropometric measure evaluated. Specifically, among women without pregnancy complications, total cholesterol was consistently associated with $\mathrm{Pl}$ at all three measurements across gestation; a 1 unit increase in cholesterol increased the PI by 2.23 to $2.55 \mathrm{~kg} \mathrm{~m}^{-3}$ independent of maternal factors. HDL was associated with decreased length at baseline and near delivery. Other associations at single time points were also observed but no associations were detected with Lp(a).

Among women with GDM, only lipid measures taken late in pregnancy were associated with neonatal size. In particular, HDL was associated with a lower birth weight $z$-score $(\beta=-1.16,95 \%$ $\mathrm{Cl}$ : $-1.92,-0.40)$, whereas triglycerides were associated with 
higher birth weight $z$-score $(\beta=0.21,95 \% \mathrm{Cl}: 0.0010,0.42)$. Among women with gestational hypertension, triglycerides were consistently associated with birth weight $z$-score regardless of time of measurement $(\beta=0.24$ to 0.31$)$. Total cholesterol and LDL were also associated with greater size measures. Among women with preeclampsia, second and third HDL measurements were associated with decreased birth weight $z$-score $(\beta=-0.49$ to -0.82$)$ and decreased PI $\left(\beta=-1.50\right.$ to $\left.-2.56 \mathrm{~kg} \mathrm{~m}^{-3}\right)$, whereas only the third measurement was associated with head circumference $(\beta=-1.33 \mathrm{~cm})$. Other sporadic associations were noted with LDL and $\operatorname{Lp}(\mathrm{a})$. Very few associations were detected between maternal lipid biomarkers and neonatal anthropometrics among women with AGT. Only HDL at the first measurement was associated with head circumference $(\beta=1.28 \mathrm{~cm} ; 95 \% \mathrm{Cl}: 0.10,2.47)$, whereas HDL at the second measurement was associated with decreased PI $\left(\beta=-3.00 \mathrm{~kg} \mathrm{~m}^{-3} ; 95 \% \mathrm{Cl}:-5.90,-0.11\right)$ (data not shown). When we examined maternal hypertriglyceridemia (triglyceride levels $>75$ th percentile value; $2.69 \mathrm{mmol} \mathrm{I}^{-1}$ ) in association with LGA, we found no significant associations in any of the pregnancy complication groups.

Given the similarities in patterns of change for each lipid measured between women with different pregnancy complications (Table 2), we examined the change in lipid levels grouping all women but also additionally adjusting for the type of pregnancy complication. Examining the longitudinal lipid measurements with the neonatal anthropometric measures shows that a $0.0037 \mathrm{mmol} \mathrm{I}^{-1}$ increase in HDL across pregnancy was associated with decreased birth weight $z$-score $(\beta=-0.22,95 \% \mathrm{Cl}$ : -0.30 , - 0.14), length $(\beta=-0.24 \mathrm{~cm}, 95 \% \mathrm{Cl}:-0.46,-0.015)$ and head circumference $(\beta=-0.24 \mathrm{~cm}, 95 \% \mathrm{Cl}:-0.38,-0.11)$, whereas a 0.028 increase in triglycerides was associated with increased birth weight $z$-score $(\beta=0.13,95 \% \mathrm{Cl}: 0.034,0.22)$ and head circumference $(\beta=0.19 \mathrm{~cm}, 95 \% \mathrm{Cl}: 0.040,0.34)$. Adjusting for pregnancy complications did not change any of the estimates. None of the other trajectories of total cholesterol, LDL and $L p(a)$ were associated with neonatal anthropometric measures (data not shown).

\section{DISCUSSION}

Main findings

We examined the dynamic changes of lipid levels across gestation and the cross-sectional associations between maternal fuels measured on average at three time points during pregnancy and neonatal anthropometrics among women with uncomplicated and complicated pregnancies. Cross-sectional associations varied by pregnancy complication group and the timing of the measurement, which may be a function of whether the measurement was taken before or after the clinical diagnosis of the complication and sample sizes of each group. However, in general, total cholesterol and triglycerides levels promoted growth while HDL levels were associated with smaller size. Women with GDM or AGT had very minimal associations between maternal lipid levels and neonatal anthropometrics, suggesting that glucose levels or other factors may have a more dominant impact on newborn size. Examining the longitudinal associations between lipids and neonatal anthropometrics after adjusting for pregnancy complications shows smaller size with increasing HDL levels and a higher birth weight $z$-score with increasing triglycerides across pregnancy.

\section{Strengths and limitations}

Strengths of our current study include the different groups of pregnancy complications that were examined and the measurements at different time points. We also add to the previous literature by examining the PI instead of birth weight alone. It has been postulated that compared to birth weight, the PI might be a better marker of future cardiovascular health as it reflects different temporal patterns of fetal undernutrition. ${ }^{19}$ Our study was limited by the lack of fasting blood samples. However, total and HDL cholesterol levels were unlikely severely impacted by fasting status. $^{20}$ Fasting time would not be associated with either neonatal anthropometry or complication status, but such random error may have reduced associations to the null. Calcium for Preeclampsia Prevention also recruited nulliparous women, who were young and with the majority being African American, which is not representative of all US pregnancies. BMI was also measured in early pregnancy.

\section{Interpretation}

The evidence of the impact of lipid levels on newborn size among women with no pregnancy complications has been inconsistent with some suggestion of differences due to pre-pregnancy BMI. One study found that triglycerides were associated with higher birth weight adjusted for gestational age but only among normal weight women, whereas HDL was associated with inverse size but only among overweight/obese women. ${ }^{21}$ On the other hand, the Pregnancy Outcomes and Community Health study $(n=1207)$ found that total cholesterol was associated with birth size in normal weight women but HDL and triglycerides in overweight/ obese women (adjusting for pregnancy complications). ${ }^{9}$ As there was inconsistent evidence and our sample size for noncomplicated women was small, we adjusted rather than stratified by BMI among our study controls. Even so, associations differed slightly also by timing of measurement and we only observed a consistent association between PI and total cholesterol, and an inverse association between length and HDL. Among undernourished Indian women, total cholesterol measured at 18 and 28 weeks' gestation was associated with birth weight independent of maternal factors such as dietary intake and other maternal fuels including fasting glucose, HDL and triglyceride. ${ }^{22}$ In a Norwegian study, an inverse association was observed with $\mathrm{HDL}$, which persisted even after adjusting for gestational weight gain, whereas a positive association between triglycerides and skinfolds (but not birth weight) was found but only after excluding women diagnosed with GDM. ${ }^{23}$ Yet, other studies have shown more consistent associations between birth weight or LGA and triglycerides but not with total cholesterol. ${ }^{24,25}$ These inconsistent associations across studies suggest that lipid measurements are unlikely to add sufficient evidence to inform practice with regards to fetal growth. HDL, which is not as subject to differences in fasting status, ${ }^{20}$ might have been a good candidate lipid biomarker but even this measure did not have consistent associations as reviewed above. Perhaps what is more important is the trajectory of $\mathrm{HDL}$, which normally decreases during pregnancy. We find that when HDL does not decrease as it should, it may be an indicator of smaller size at birth as observed in our longitudinal models.

In our examination of women with hypertensive disorders, we found different results depending on the severity of hypertension and timing of measurement. As Calcium for Preeclampsia Prevention was a trial of preeclampsia, women with blood pressure $>135 / 85 \mathrm{~mm} \mathrm{Hg}$ were excluded from participation. ${ }^{12}$ As such, lipid measures at baseline reflect values bfore clinical diagnosis of hypertension or preeclampsia. These associations, however, still differed from the control group and only among women with gestational hypertension were consistent associations observed between triglycerides and the birth weight $z$-score. Although studies have noted that women with gestational hypertension have higher triglyceride levels than women without gestational hypertension, ${ }^{26}$ no previous studies to our knowledge have examined how maternal lipid biomarkers are associated with newborn anthropometrics among women with gestational hypertension. Interestingly, only women with preeclampsia 
showed associations between $L p(a)$ and two of the examined newborn measures including birth weight $z$-score and length. Worth noting is that one third of the women with preeclampsia delivered a preterm infant.

Lp(a), a carrier of cholesterol, has been associated with increased risk of a variety of cardiovascular disorders. ${ }^{27}$ As preeclampsia has some features of coronary heart disease, several studies have addressed the role of $L p(a)$ in preeclampsia. ${ }^{28}$ The role of $\mathrm{Lp}(\mathrm{a})$ in either normal or complicated pregnancies has been less conclusive. Two meta-analyses concluded that the equivocal evidence has been a result of the different methods used in measuring $L p(a)$, the sample size, study design and ethnicity of the study population. ${ }^{28,29}$ To our knowledge, the $L p(a)$ association with neonatal anthropometrics has not been explored previously.

The minimal associations observed for women with GDM or AGT might be due to the overwhelming effects of plasma glucose. ${ }^{30}$ In a study of over 2200 mother-baby pairs (20\% with GDM), first visit fasting plasma glucose (10 to 24 weeks' gestation) was associated with birth weight and birth length. ${ }^{4}$ In a subsequent study among over 1500 mother-baby pairs by the same group, first visit triglycerides were also positively associated with birth weight and head circumference. ${ }^{8}$ Contrary to our findings for the AGT group, two previous studies of nondiabetic women ( $n=146$ and $n=83$ ) with a positive diabetic screen found associations between birth weight and triglyceride levels. ${ }^{5,6}$ Both studies further found that maternal hypertriglyceridemia, defined as triglyceride levels above the 75th percentile, was associated with having an LGA infant at term ${ }^{5,6}$ with one study showing hypertriglyceridemia $>259 \mathrm{mg} \mathrm{dl}^{-1}$ (odds ratio $=11.6 ; 95 \% \mathrm{Cl}: 1.1$, 122) to be an independent predictor of LGA after accounting for other maternal factors including prepregnancy BMI and fasting plasma glucose levels. ${ }^{6}$ The lack of association with LGA in our study might be due to sample size limitations as we only had 67 women with AGT measured between 22 and 32 weeks' gestation with only 11 women having triglyceride levels above $259 \mathrm{mg} \mathrm{dl}^{-1}$. Similarly, defining hypertriglyceridemia as the 75th cutpoint for our sample did not impact the results (data not shown). Among women with $\operatorname{GDM}(n=150)$, maternal triglycerides measured during the 3rd trimester correlated significantly with newborn fat mass ( $r=0.17, P=0.03$ ) but not with birth weight or neonatal BMI. ${ }^{11}$ In addition, maternal triglycerides at delivery remained associated with LGA independent of maternal BMI. ${ }^{11}$ Associations between maternal serum triglyceride levels measured in early pregnancy and at 24 to 28 weeks' gestation and birth weight ratio (birth weight corrected for gestational age) independent of maternal factors have been reported in other studies. ${ }^{7,10}$

\section{CONCLUSION}

Despite general observations that HDL was associated with reduced neonatal size, while total cholesterol and triglycerides may be associated with larger size, our findings in combination with evidence from the literature suggest that lipid biomarkers are not likely to serve as clinically useful markers of fetal growth and are unlikely to change clinical decision making. The onset of pregnancy complications, especially GDM and preeclampsia, appeared to further deteriorate the ability of the lipid biomarkers to predict neonatal size. Pooling individual data across studies may serve as one way to tease apart the utility of these markers. All data from this study are being made available to further such investigations.

\section{CONFLICT OF INTEREST}

$\mathrm{PM}, \mathrm{AL}$ and $\mathrm{EHY}$ are employees of the federal government All other authors declare no conflicts of interest.

\section{ACKNOWLEDGEMENTS}

This study was supported by the Intramural Research Program of the Eunice Kennedy Shriver National Institute of Child Health and Human Development. The Calcium for Preeclampsia Prevention trial was originally supported by contracts (N01-HD-1-3121, $-3122,-3123,-3124,-3125$ and -3126; N01-HD-3154; and N01-HD-5-3246) with the National Institute of Child Health and Human Development, with co-funding from the National Heart, Lung, and Blood Institute. Additional biomarker assays including for lipids was supported by contract (HHSN275201100002I-HHSN27500003) with the Eunice Kennedy Shriver National Institute of Child Health and Human Development.

\section{REFERENCES}

1 Barker DJ. The developmental origins of chronic adult disease. Acta Paediatr Suppl 2004; 93(446): 26-33.

2 Metzger BE, Lowe LP, Dyer AR, Trimble ER, Chaovarindr U, Coustan DR et al. Hyperglycemia and adverse pregnancy outcomes. N Engl J Med 2008; 358(19): 1991-2002.

3 Jovanovic L, Pettitt DJ. Gestational diabetes mellitus. JAMA 2001; 286(20): 2516-2518.

4 Liu B, Chen H, Xu Y, An C, Zhong L, Wang X et al. Fetal growth is associated with maternal fasting plasma glucose at first prenatal visit. PLOS ONE 2014; 9(12): e116352

5 Di CG, Miccoli R, Volpe L, Lencioni C, Ghio A, Giovannitti MG et al. Maternal triglyceride levels and newborn weight in pregnant women with normal glucose tolerance. Diabet Med 2005; 22(1): 21-25.

6 Kitajima M, Oka S, Yasuhi I, Fukuda M, Rii Y, Ishimaru T. Maternal serum triglyceride at 24-32 weeks' gestation and newborn weight in nondiabetic women with positive diabetic screens. Obstet Gynecol 2001; 97(5 Pt 1): 776-780.

7 Knopp RH, Magee MS, Walden CE, Bonet B, Benedetti TJ. Prediction of infant birth weight by GDM screening tests. Importance of plasma triglyceride. Diabetes Care 1992; 15(11): 1605-1613.

8 Liu B, Geng H, Yang J, Zhang Y, Deng L, Chen W et al. Early pregnancy fasting plasma glucose and lipid concentrations in pregnancy and association to offspring size: a retrospective cohort study. BMC Pregnancy Childbirth 2016; 16: 56.

9 Mudd LM, Holzman CB, Evans RW. Maternal mid-pregnancy lipids and birthweight. Acta Obstet Gynecol Scand 2015; 94(8): 852-860.

10 Nolan CJ, Riley SF, Sheedy MT, Walstab JE, Beischer NA. Maternal serum triglyceride, glucose tolerance, and neonatal birth weight ratio in pregnancy. Diabetes Care 1995; 18(12): 1550-1556.

11 Schaefer-Graf UM, Graf K, Kulbacka I, Kjos SL, Dudenhausen J, Vetter K et al. Maternal lipids as strong determinants of fetal environment and growth in pregnancies with gestational diabetes mellitus. Diabetes Care 2008; 31(9): 1858-1863.

12 Levine RJ, Esterlitz JR, Raymond EG, DerSimonian R, Hauth JC, Ben CL et al. Trial of Calcium for Preeclampsia Prevention (CPEP): rationale, design, and methods. Control Clin Trials 1996; 17(5): 442-469.

13 Levine RJ, Hauth JC, Curet LB, Sibai BM, Catalano PM, Morris CD et al. Trial of calcium to prevent preeclampsia. N Engl J Med 1997; 337(2): 69-76.

14 Levine RJ, Lam C, Qian C, Yu KF, Maynard SE, Sachs BP et al. Soluble endoglin and other circulating antiangiogenic factors in preeclampsia. N Engl J Med 2006; 355(10): 992-1005

15 Yeung EH, Liu A, Mills JL, Zhang C, Mannisto T, Lu Z et al. Increased levels of copeptin before clinical diagnosis of preelcampsia. Hypertension 2014; 64(6): 1362-1367.

16 Joffe GM, Esterlitz JR, Levine RJ, Clemens JD, Ewell MG, Sibai BM et al. The relationship between abnormal glucose tolerance and hypertensive disorders of pregnancy in healthy nulliparous women. Calcium for Preeclampsia Prevention (CPEP) Study Group. Am J Obstet Gynecol 1998; 179(4): 1032-1037.

17 American Diabetes Association. Diagnosis and classification of diabetes mellitus. Diabetes Care 2011; 34(Suppl 1): S62-S69.

18 Brenner WE, Edelman DA, Hendricks $\mathrm{CH}$. A standard of fetal growth for the United States of America. Am J Obstet Gynecol 1976; 126(5): 555-564.

19 Huxley R, Owen CG, Whincup PH, Cook DG, Rich-Edwards J, Smith GD et al. Is birth weight a risk factor for ischemic heart disease in later life? Am J Clin Nutr 2007; 85(5): 1244-50.

20 Craig SR, Amin RV, Russell DW, Paradise NF. Blood cholesterol screening influence of fasting state on cholesterol results and management decisions. J Gen Intern Med 2000; 15(6): 395-399.

21 Misra VK, Trudeau S, Perni U. Maternal serum lipids during pregnancy and infant birth weight: the influence of prepregnancy BMI. Obesity (Silver Spring) 2011; 19(7): 1476-1481.

22 Kulkarni SR, Kumaran K, Rao SR, Chougule SD, Deokar TM, Bhalerao AJ et al. Maternal lipids are as important as glucose for fetal growth: findings from the Pune Maternal Nutrition Study. Diabetes Care 2013; 36(9): 2706-2713. 
23 Sommer C, Sletner L, Morkrid K, Jenum AK, Birkeland KI. Effects of early pregnancy $\mathrm{BMI}$, mid-gestational weight gain, glucose and lipid levels in pregnancy on offspring's birth weight and subcutaneous fat: a population-based cohort study. BMC Pregnancy Childbirth 2015; 15: 84.

24 Vrijkotte TG, Algera SJ, Brouwer IA, van EM, Twickler MB. Maternal triglyceride levels during early pregnancy are associated with birth weight and postnatal growth. J Pediatr 2011; 159(5): 736-742.

25 Jin WY, Lin SL, Hou RL, Chen XY, Han T, Jin $Y$ et al. Associations between maternal lipid profile and pregnancy complications and perinatal outcomes: a population-based study from China. BMC Pregnancy Childbirth 2016; 16: 60 .

26 Vrijkotte TG, Krukziener N, Hutten BA, Vollebregt KC, van EM, Twickler MB Maternal lipid profile during early pregnancy and pregnancy complications and outcomes: the ABCD study. J Clin Endocrinol Metab 2012; 97(11): 3917-3925.

27 Marcovina SM, Koschinsky ML, Albers JJ, Skarlatos S. Report of the National Heart, Lung, and Blood Institute Workshop on Lipoprotein(a) and Cardiovascular Disease: recent advances and future directions. Clin Chem 2003; 49(11): 1785-1796.

28 Fanshawe $A E$, Ibrahim $M$. The current status of lipoprotein (a) in pregnancy: a literature review. J Cardiol 2013; 61(2): 99-106.

29 Manten GT, Voorbij HA, Hameeteman TM, Visser GH, Franx A. Lipoprotein (a) in pregnancy: a critical review of the literature. Eur J Obstet Gynecol Reprod Biol 2005; 122(1): 13-21.

30 Wahabi HA, Fayed AA, Alzeidan RA, Mandil AA. The independent effects of maternal obesity and gestational diabetes on the pregnancy outcomes. BMC Endocr Disord 2014; 14: 47. 